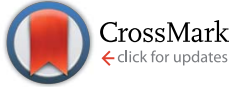

Cite this: RSC Adv., 2017, 7, 3190

Received 24th October 2016 Accepted 5th December 2016 DOI: 10.1039/c6ra25771b www.rsc.org/advances

\section{Electrochemical and electromechanical behavior of Nafion-based soft actuators with PPy/CB/MWCNT nanocomposite electrodes}

\begin{abstract}
Haleh Rasouli, ${ }^{a}$ Leila Naji*a and Mir Ghasem Hosseini ${ }^{\mathrm{b}}$
In this work, as an alternative to precious platinum electrodes in IPMC actuators, PPy/CB/MWCNT electrode actuators were successfully fabricated by electropolymerization of PPy on both sides of the CB/MWCNTcoated Nafion membranes. Electropolymerization of PPy was performed using cyclic voltammetry (CV) and chronopotentiometry (CHP) in water and acetonitrile (AN) to study the effect of the synthesis parameters on the physical, electrochemical and electromechanical characteristics of actuators. The electrochemical characteristics of the actuators were investigated using cyclic voltammetry (CV) and electrochemical impedance spectroscopy (EIS). The physical and structural properties of the actuators were characterized using scanning electron microscopy (SEM), water uptake (WUP) and ion exchange capacity (IEC) determination methods. SEM analysis revealed that the surface morphology of actuators changes considerably by altering the synthesis parameters and that the finest cauliflower-like structure of the PPy film is formed in actuators prepared in AN using the CHP technique. These metal-free actuators showed ionic conductivity $\left(29.88 \mathrm{mS} \mathrm{cm} \mathrm{cm}^{-1}\right)$ and capacitive characteristics $(39.23 \mu \mathrm{F})$ about eleven and thirty six times higher than that considered for Pt-based IPMC actuators, respectively. The deposition of PPy/CB/ MWCNT electrodes on the Nafion membrane also resulted in higher WUP (79.9\%) and IEC $\left(6.7 \mathrm{meq} \mathrm{g}^{-1}\right)$ of the actuators, confirming the hydrophilic nature of the PPy-based electrodes. The maximum tip displacement of these actuators (at optimized conditions) was however about $26 \%$ lower than that of Ptbased IPMC actuators which can be improved by decreasing the thickness and surface resistance of the PPy film via altering the synthesis parameters.
\end{abstract}

\section{Introduction}

Ionic polymer-metal composites (IPMCs) ${ }^{\mathbf{1}}$ which are classified as ionic electroactive polymers (EAPs) exhibit bending deformation when a small electrical potential (1-5 V) is applied across their thickness. The fascinating characteristics of IPMCs including softness, lightweight, biocompatibility, flexibility and large displacement ${ }^{2}$ have made them an attractive research subject for use in a wide range of industrial and medical applications as soft actuators. $^{3-5}$ An IPMC actuator is typically composed of a thin cation-exchange polymer membrane (mostly Nafion), through which ions of single or multiple charge can pass, and a pair of electrodes (generally $\mathrm{Pt}$ or $\mathrm{Au}$ ) which are chemically plated on both surfaces. ${ }^{6,7}$ Prior to use, the IPMC is soaked in an aqueous ionic solution to hydrate the polymer and to allow the exchange of the desired cation ( $\mathrm{Li}^{+}$in this work). In the absence of an applied potential, the anionic groups $\left(-\mathrm{SO}_{3}{ }^{-}\right.$group, in Nafion) are

${ }^{a}$ Department of Chemistry, AmirKabir University of Technology, 424 Hafez Avenue, Tehran, 15875-4413, Iran. E-mail: leilanaji@aut.ac.ir; Fax: +98 21 64542762; Tel: +982164542767

${ }^{b}$ Electrochemistry Research Laboratory, Department of Physical Chemistry, Chemistry Faculty, University of Tabriz, Tabriz, Iran attached to the polymer backbone and the cations are associated. When a potential is applied, the anionic groups are unable to move far towards the positively charged anode, whereas the cations are able to break away from the anionic groups and move towards the negative cathode, forming volumetric gradient or pressure gradient between electrodes on both sides. This gradient is responsible for bending motion of the IPMC actuator towards anode. ${ }^{8}$ Therefore, IPMCs store electrical energy in a double interface and convert it into mechanical output by reversible ion intercalation and de-intercalation of electrodes., ${ }^{\mathbf{9 1 0}}$ Ion exchange membranes, ${ }^{11}$ the charge and mobility of the cations ${ }^{12}$ the solvent content of the polymer, ${ }^{13}$ the structure and capacitance of the electrodes ${ }^{\mathbf{1 4}}$ and the specific interactions between the electrode material and the cations ${ }^{\mathbf{1 2 , 1 5}}$ are significant factors that largely influence the actuation performance of IPMCs. Under long-term actuation in open air, however, Nafion-based IPMC actuators become dehydrated due to the leakage of the inner electrolyte and hydrated cations through cracks in the metallic electrodes. ${ }^{\mathbf{1 6}}$ There have been many studies to overcome the mentioned drawbacks by modifying the ion exchange membrane, ${ }^{17,18}$ replacing water with non-volatile solvents, ${ }^{16}$ and replacing conventional metal electrodes with flexible conducting materials. In previous works, we illustrated that the incorporation of $\mathrm{Li}^{+}$ions 
to the ionic liquid-based IPMCs ${ }^{12}$ and also the addition of sulfonated graphene oxide to Nafion membrane ${ }^{16}$ promoted the actuation response of Pt-based IPMC actuators by enhancing the capacitance, ionic conduction, operating life and the displacement rate of the actuator.

It has been established that the actuation performance of IPMC actuators is mainly dominated by electrochemical and electromechanical process of the electrode layer. ${ }^{19,20}$ Thus, the electrode material and the electrode structure become more crucial to achieve actuators with high performances. Surely, electrode materials with lower cost, higher flexibility and appropriate electrochemical and electromechanical properties are of great importance for various potential applications. Researchers have shown that introducing carbon nanostructures ${ }^{21}$ into the electrode layer results in a significant improvement of the actuation performance of the IPMC actuators. ${ }^{22,23}$ Carbon nanotubes $(\mathrm{CNTs})^{24}$ and carbon black (CB) ${ }^{21}$ possess high surface area, and exceptional electrical, mechanical and chemical characteristics and their application in electrochemical double-layer capacitors has been studied in detail. Investigating polymer-based actuators containing single-wall carbon nanotubes (SWCNTs), ${ }^{25}$ activated multi-walled carbon nanotubes (MWCNTs), ${ }^{26}$ and a combination of non-activated MWCNTs and a metal oxide ${ }^{27}$ showed that by introducing carbon nanostructures into the electrode, the actuation performances including respond speed, actuation displacement and strain rate could be significantly improved. ${ }^{20}$ The volume change of carbon nanostructures-electrode based ionic actuators is caused by the intercalation of ions due to the electric double layer charging of the electrodes. ${ }^{28,29}$ However, carbon nanostructures have low capacitance which can be improved by compositing with other conducting materials. ${ }^{30,31}$ Conducting polymers (CPs) are soft actuation and sensing materials with promising applications in biomedical devices ${ }^{32}$ and micromanipulation systems ${ }^{33}$ due to their good electrical conductivity, large pseudo-capacitance, and relatively low cost. The most commonly used CPs include polyaniline (PANI), ${ }^{34}$ polypyrrole $(\mathrm{PPy})^{35,36}$ and poly[3,4-ethylenedioxythiophene] (PEDOT) ${ }^{37}$ CPs have been combined with carbon nanostructures ${ }^{38-40}$ and characterized for electromechanical actuator applications ${ }^{41}$ and electrochemical capacitors. ${ }^{42,43}$ These composites are interesting since they can combine two relatively low-cost materials to gain the large pseudo-capacitance of the CPs coupled with the conductivity and mechanical strength of the carbon nanostructures. The volume change of CPs is caused by insertion and extraction of ions and solvent from the electrolyte when material is electrochemically switched between the oxidized and reduced states. Mostly, PPy is used as actuator material since it is easy to fabricate and actuate and it possesses high conductivity, environmental stability and ability to form freestanding films ${ }^{44,45}$ For actuators, electrochemical polymerization is most commonly utilized to prepare PPy film since it is a stable and well-reproducible synthetic method and can rapidly synthesize polymer film with different properties by adjusting the procedure parameters. ${ }^{46,47}$ In general, galvanostatic or potentiostatic method is applied to synthesize the CPs, although more elaborate deposition methods have been reported, such as a sequence of potential steps. ${ }^{44}$ The choice of solvent in electropolymerization method is of critical importance, determining both the physical characteristic (i.e. porosity and conductivity) and the actuation performance of CPs.

Due to the hydrophobic nature and strong van der Waals interactions between MWCNTs, they intend to pack into crystalline ropes and tangle networks. This diminishes the special mechanical and electrical properties of the individual tubes. ${ }^{48}$ In this work, we constructed novel metal-free ionic actuators by sandwiching Nafion-117 membrane between two pieces of CB/ MWCNT/PPy nanocomposite film based electrode, in which the PPy films were uniformly deposited onto the CB/MWCNT-coated Nafion membrane through electropolymerization method. A combination of the electrical conductivity, large surface area of the individual $\mathrm{CB}$ and MWCNT nanoparticles and excellent electrochemical properties of the PPy films resulted in the composite electrode having good physical properties and a high electrochemical capacitance. The properties of PPy film and the electromechanical performance of resulting actuators were manipulated by altering the electropolymerization parameters; including solvent type and potential regime. To this end, electropolymerization of PPy was performed using cyclic voltammetry (CV) and chronopotentiometry (CHP) in water and acetonitrile (AN). The effects of the above-mentioned parameters were followed using physical, electrochemical and electromechanical measurements and were compared with the corresponding behavior of Pt electrode-based IPMC actuators. SEM analysis revealed that the surface morphology of actuators changes considerably by altering the synthesis parameters and that the finest cauliflower-like structure of electrode layer forms in actuators prepared in AN using CHP technique. These actuators showed the highest water uptake (WUP), ion exchange capacity (IEC), hydrated thickness, electrode capacitance, ionic conduction and tip displacement. The actuation performance of the fabricated metal-free actuators was comparable to the conventional Pt-electrode based IPMC actuators.

\section{Experimental}

\subsection{Materials}

Pyrrole (98\%) and acetonitrile (AN, 99.9\%) were acquired from Merck and vacuum-distilled prior to use. Lithium chloride (LiCl, $99 \%)$ and oxalic acid $\left(\mathrm{H}_{2} \mathrm{C}_{2} \mathrm{O}_{4}, 2 \mathrm{H}_{2} \mathrm{O}, 99 \%\right)$ were obtained from Fluka and used as received. Isopropyl alcohol $\left(\mathrm{C}_{3} \mathrm{H}_{8} \mathrm{O}, 99 \%\right)$ and nitric acid $\left(\mathrm{HNO}_{3}, 65 \%\right)$ were received from Merck. Distilled water, activated CB, Vulcan XC-72R carbon, was purchased from Cabot Company. Functionalized multi walled carbon nanotubes (MWCNTs, 99\%) was purchased from Sigma Aldrich ( $\mathrm{COOH}$ content $8 \mathrm{wt} \%$ ). DuPont liquid Nafion solution (5 wt\%), in a mixture of lower aliphatic alcohols and water (45 wt\%), with an equivalent weight of 1100 and Nafion 117 sheet of thickness 0.18 $\mathrm{mm}$ in protonated form were purchased from Aldrich.

\subsection{Preparation of $\mathrm{CB} / \mathrm{MWCNT}$-coated Nafion membrane}

To prepare CB/MWCNT ink $60 \mathrm{mg}$ Vulcan XC-72R carbon, $30 \mathrm{mg}$ MWCNT and $30 \mathrm{mg}$ of Nafion solution $5 \mathrm{wt} \%$ was added in $20 \mathrm{~mL}$ mixture of isopropanol-DI water $(3: 1 \mathrm{v} / \mathrm{v})$. The mixture was then ultrasonicated for $1.5 \mathrm{~h}$ and stirred at $2000 \mathrm{rpm}$ for $24 \mathrm{~h}$. The 


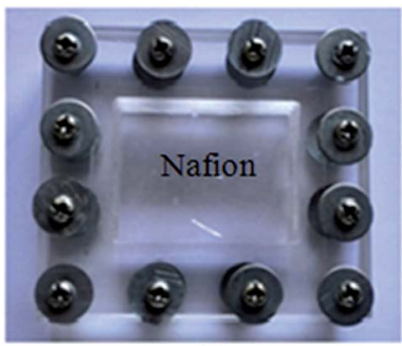

(a)

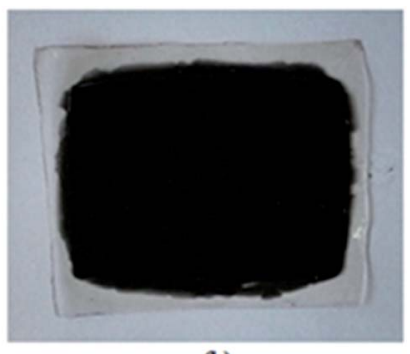

(b)
Fig. 1 Digital images of (a) Nafion membrane in the Plexiglas fram, (b) CB/MWCNT-coated Nafion membrane.

Nafion solution in the prepared ink serves as the binder and ion conductor and provides a suitable interface between the Nafion membrane and electrode materials.

A $3 \times 4 \mathrm{~cm}$ sample of commercial Nafion membrane was roughened at both surfaces using abrasive paper to increase the electrode-polymer interface area. To remove all impurities remaining from the manufacturing process the membrane was washed using an extensive cleaning process described elsewhere. ${ }^{\mathbf{1 2 , 4 9}}$ The pre-cleaned Nafion membrane was placed between two sintered glass plates (to avoid membrane deformation during drying) and put in an oven at $70{ }^{\circ} \mathrm{C}$ for $1 \mathrm{~h}$. Nafion membrane have a tendency to curl or be pushed sideways when absorb water or other solvents. This limits the accuracy of the ink coating process. To avoid the effects of Nafion membrane deformation, the membrane was mounted in a $3 \mathrm{~cm} \times 4 \mathrm{~cm}$ Plexiglas holder shown in Fig. 1(a) and then put on the hot plate at $60{ }^{\circ} \mathrm{C}$ prior to ink coating process. A same volume of the prepared $\mathrm{CB} / \mathrm{MWCNT}$ ink was carefully sprayed on both sides of the membrane using a jet spray. The CB/MWCNT-coated membrane was then placed in an oven at $80{ }^{\circ} \mathrm{C}$ for $15 \mathrm{~min}$ to remove excess solvents. The $\mathrm{CB} / \mathrm{MWCNT}$-coated Nafion membrane (shown in Fig. 1(b)) was cut in $3 \mathrm{~cm} \times 0.5 \mathrm{~cm}$ strips and stored in DI water for use in actuator preparation.

\subsection{Electrodeposition of PPy films}

To prepare the actuators, PPy was electrochemically grown on both faces of the CB/MWCNT-coated Nafion membrane immersed as anode in solutions containing $0.1 \mathrm{M}$ pyrrole and $0.1 \mathrm{M} \mathrm{LiCl}$. These experiments were carried out in a conventional three-electrode cell, using platinum plate and saturated calomel electrode (SCE) as counter and reference electrodes, respectively. Prior to the experiment, the electrolyte solution was purged with nitrogen to remove oxygen, because films prepared in the presence of oxygen have inferior properties. It has been established that the mechanical properties of the PPy film, their morphology and electrochemical behavior depend on the conditions of preparation, including the applied potential regime, the nature of solvent, the purity and concentration of the initial monomer, and the nature and concentration of electrolytic salt. ${ }^{50}$ Therefore, electropolymerization of pyrrole was performed using cyclic voltammetry (CV) and chronopotentiometry (CHP) in water and a mixture of acetonitrile-water $(97: 3 \mathrm{v} / \mathrm{v})$ to study the effect of these parameters on the physical, electrochemical and electromechanical characteristic of the actuators. According to the extensively used procedure of Diaz, ${ }^{50}$ adding a small amount of water to acetonitrile solution results in improved properties (adhesion to electrode, uniformity) of PPy film. The mixture of acetonitrile-water was named as AN in this text. Fig. 2 illustrates the fabrication process of PPy-electrode actuators. The electropolymerization on the $\mathrm{CB} / \mathrm{MWCNT}$-coated membrane was performed by continuous potential cycling at a sweep rate of $0.03 \mathrm{~V}$ $\mathrm{s}^{-1}$ and in the potential range of -0.2 to $1 \mathrm{~V}$ through 35 scans. The PPy synthesis on the CB/MWCNT-coated membrane was also achieved using CHP technique in similar solutions, at current density of $2 \mathrm{~mA} \mathrm{~cm}^{-2}$ and duration of $3600 \mathrm{~s}$. The total electropolymerization time was calculated according to the particular current density to ensure that the total charge passed was the same for each sample. All experiments were performed on a potentiostat/galvanostat (Autolab) controlled by NOVA software at ambient temperature. The membranes were then rinsed with ethanol and DI water repeatedly, immersed in $1 \mathrm{M} \mathrm{HNO}_{3}$ solution

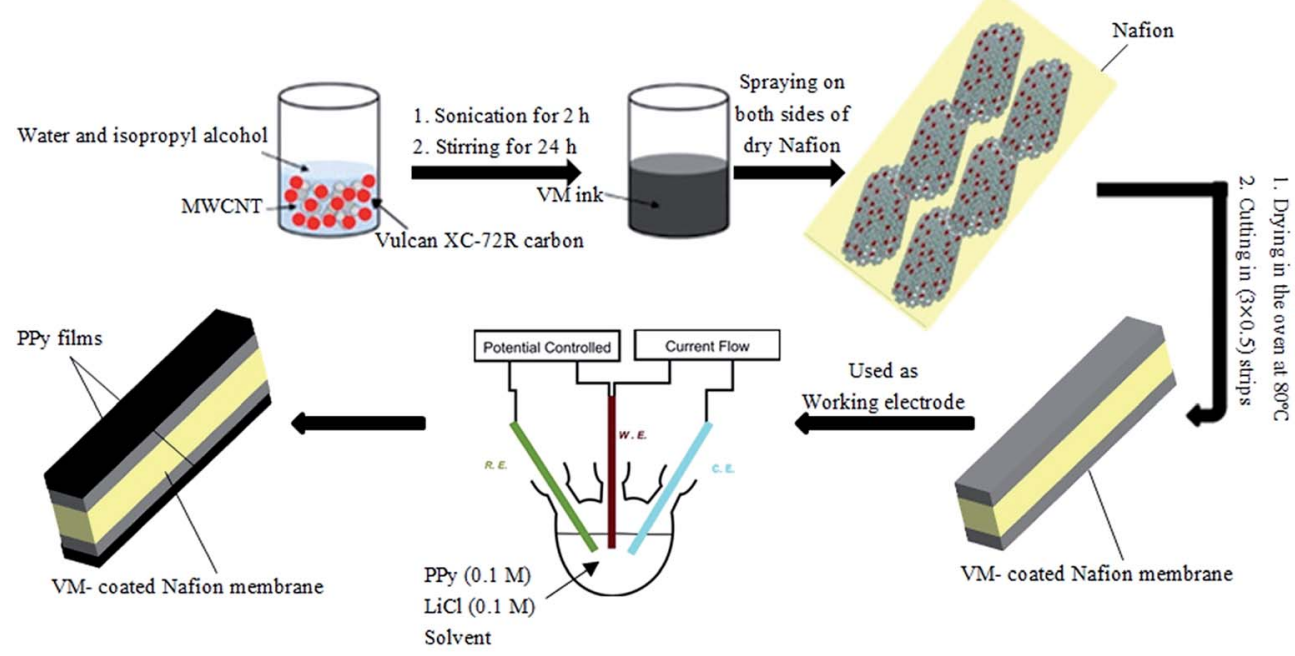

Fig. 2 Schematic diagram of the fabrication process of PPy/CB/MWCNT electrode-based actuators. 
Table 1 Naming system of actuators

\begin{tabular}{lllll}
\hline Sample name & LiCl (M) & Pyrrole (M) & Solvent & Synthesis method \\
\hline IP & - & - & - & - \\
IP-W-CV & 0.1 & 0.1 & $\mathrm{H}_{2} \mathrm{O}$ & CV \\
IP-W-CHP & 0.1 & 0.1 & $\mathrm{H}_{2} \mathrm{O}$ & $\mathrm{CHP}$ \\
IP-AN-CV & 0.1 & 0.1 & $\mathrm{AN}: \mathrm{H}_{2} \mathrm{O}$ & $\mathrm{CV}$ \\
IP-AN-CHP & 0.1 & 0.1 & $\mathrm{AN}: \mathrm{H}_{2} \mathrm{O}$ & CHP
\end{tabular}

for $12 \mathrm{~h}$, rinsed again and stored in aqueous $1 \mathrm{M} \mathrm{LiCl}$ solution to exchange all protons in polymer with $\mathrm{Li}^{+}$ions. The actuators were named to indicate the type of the solvent and electrochemical technique used in their fabrication; IP-W-CV and IP-AN-CV for actuators prepared using $\mathrm{CV}$ technique and IP-W-CHP and IP-AN$\mathrm{CV}$ for actuators prepared using CHP technique in water and AN, respectively. Table 1 gives the names and the detailed process information for all actuators. The physical, electrochemical and electromechanical behavior of the fabricated PPy/CB/MWCNTelectrode based actuators were compared with Pt-electrode based IPMC actuator prepared by depositing Pt electrodes on the faces of the cleaned Nafion membrane, using an electroless plating method described in our previous work. ${ }^{16}$ The Pt-based IPMC actuator was named as IP in this work.

\subsection{Physical and morphological characterization of actuators}

Water uptake (WUP) of the actuators was calculated using eqn (1):

$$
\% \mathrm{WUP}=100 \times\left(W_{\mathrm{wet}}-W_{\mathrm{dry}} / W_{\mathrm{dry}}\right)
$$

where $W_{\text {wet }}$ and $W_{\text {dry }}$ are wet weight and dry weight of the actuators, respectively. To determine $W_{\text {wet }}$ of the actuators, they were equilibrated in DI water at room temperature for $24 \mathrm{~h}$. The fully hydrated actuators were then removed from water and the excess water was wiped off from their surface using a tissue paper and weighted instantly. The actuators were then vacuum dried in an oven at $80{ }^{\circ} \mathrm{C}$ overnight and their $W_{\text {dry }}$ was measured.

The hydrated thickness of the actuators was measured at multiple points using a digital micrometer. The ionic-exchange capacity (IEC) of the prepared actuators was measured by classical titration method, described elsewhere. ${ }^{\mathbf{1 6}}$

Scanning electron microscopy (SEM) was employed to study the morphology of the electrode region of actuators using a TESCANMIRA3 SEM instrument. SEM was performed at an accelerating voltage of $20.0 \mathrm{kV}$ at $500 \mathrm{~nm}$ and $2 \mu \mathrm{m}$ magnification levels.

\subsection{Electrochemical characterization of actuators}

The ionic conduction and capacitive characteristics of the prepared actuators were studied using electrochemical impedance spectroscopy (EIS) and cyclic voltammetry (CV). To this end, square elements of $5 \mathrm{~mm} \times 5 \mathrm{~mm}$ were cut from the actuators and sandwiched between a pair of platinum contacts (with the same surface area) embedded within a Plexiglas clamp. The measurements were performed on a potentiostat/galvanostat (EG\&G) controlled by PowerSuite software at ambient temperature. CV scans were carried out in the potential range of -0.2 to $1 \mathrm{~V}$ at a scan rate of $0.03 \mathrm{~V} \mathrm{~s}^{-1}$. EIS measurements were performed in the frequency range of $100 \mathrm{kHz}$ to $100 \mathrm{mHz}$ by superimposing an a.c. voltage of $5 \mathrm{mV}$ and the results were analyzed using Z-view software. Proton conductivity $(\sigma)$ was obtained using eqn (2), where $\sigma$ is the proton conductivity $\left(\mathrm{S} \mathrm{cm}^{-1}\right)$, $l$ the measured thickness of the actuators $(\mathrm{cm}), R$ is the resistance $(\Omega)$ and $A$ is the area of the actuators $\left(\mathrm{cm}^{2}\right)$.

$$
\sigma=l / R A
$$

\subsection{Actuation measurements of actuators}

Maximum tip displacement of the free end of the actuators was measured by applying a d.c. voltage across the thickness of the sample under study and the effect of varying amplitude of the input voltage was investigated. These measurements were carried out in an open air environment $\left(25^{\circ} \mathrm{C}\right)$ of $40 \%$ humidity using the arrangement shown in our previous work. ${ }^{12}$ To this end, the actuator of standard dimensions of $0.5 \mathrm{~cm}$ wide by $3 \mathrm{~cm}$ long was held at one end in a sample holder consisting of a pair of platinum contacts within an electrically insulating clamp. The metal contacts in turn were connected to the two terminals of a d.c. power supply using thin wires. All actuators were subjected to d.c. voltages from 1 to $8 \mathrm{~V}$. Video images of the bending deformation of the free end of the sample were captured by the digital camera. The images were then transferred to a computer and the average maximum peak tip displacement of the actuator was determined by measuring the linear distance between the starting point and end point of the free end of the actuators, using image analysis software.

\section{Results}

\subsection{Electropolymerization of PPy}

Generally, in CHP technique, a constant current pulse is applied to the working electrode and its resulting potential is measured against the reference electrode as a function of time. Fig. 3

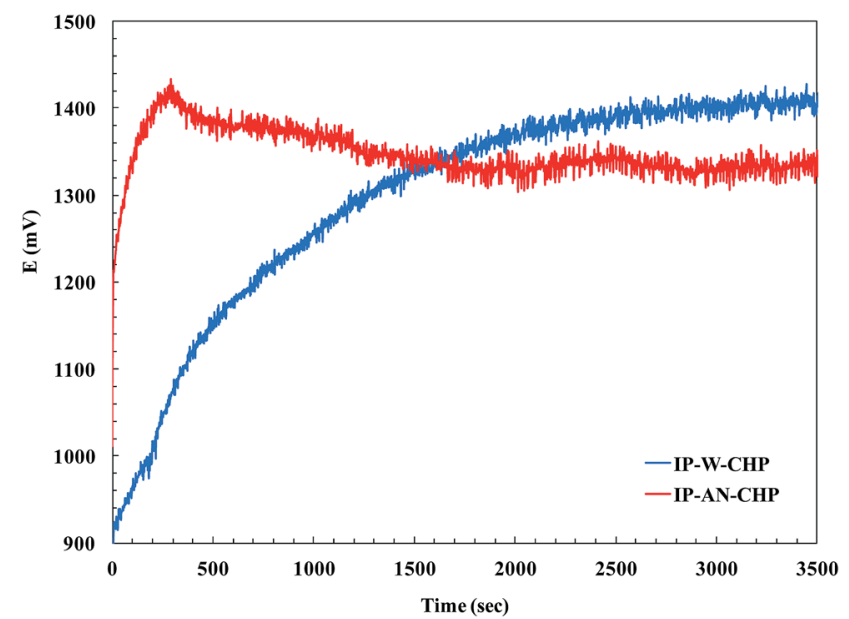

Fig. 3 The effect of solvent on the chronopotentiograms of polymerization of PPy in fabrication of IP-AN-CHP and IP-W-CHP actuators. 


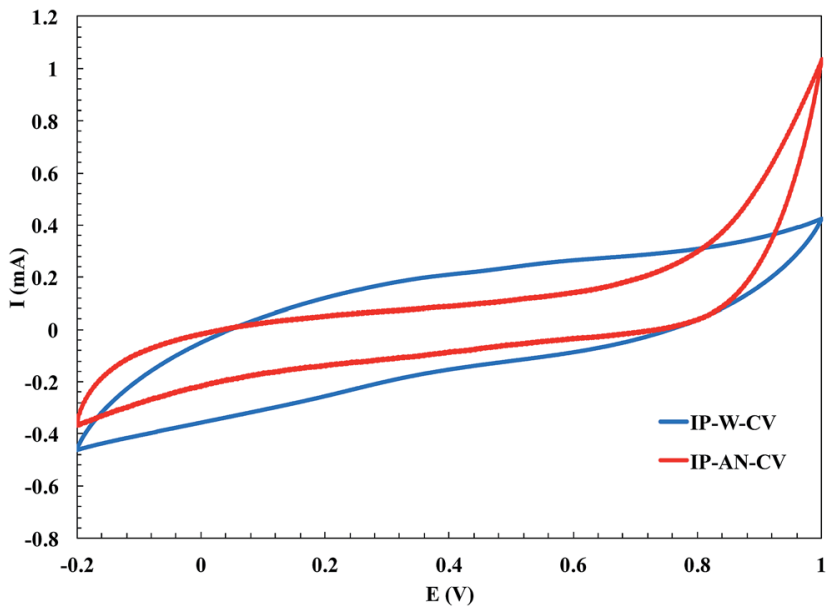

Fig. 4 The effect of solvent on the cyclic voltammograms (average of 35 cycles) of polymerization of PPy in fabrication of IP-W-CV and IPAN-CV actuators.

compares the $E-t$ curve during CHP electropolymerization of pyrrole in water and AN. In both cases, as the current was applied, the measured potential was abruptly increased due to the ohmic loss, and afterward it gradually changed and stabilized. However, the mentioned potential change occurred in a significantly shorter time in the case of electropolymerization in AN. In other words, the time elapsed for achieving to the desired anodic potential was much longer in water compared to that observed in AN, indicating a higher rate of nucleation and polymerization of PPy in AN (IP-AN-CHP actuator). This was attributed to the higher donor number of AN in comparison with water. ${ }^{50}$ The earlier electrochemical studies have reported that the first stage of the oxidation reaction of pyrrole to PPy involves the electrode oxidation of monomer yielding radical cations. ${ }^{35}$ The stability of radical cations is an important factor in electropolymerization. The radical cations with moderate stability possess the chemical selectivity which favors radical coupling in the film formation. ${ }^{51}$ However, the radical cations with lower stability are more reactive and react with solvent or other nucleophiles close to the electrode surface. Therefore, the yield of polymer and its conductivity can grow with increasing donor number of the solvent. The results shown in Fig. 3 suggest that the radical cations formed in AN possessed higher stability and could react selectively with the MWCNTs ${ }^{52,53}$ and pyrrole monomers, resulting in the faster PPy film formation. Similar trend was observed in electropolymerization of pyrrole using CV technique. Fig. 4 compares the cyclic voltammograms of electropolymerization of pyrrole on the CB/MWCNT-coated Nafion membrane in water and AN. In CV measurements, the current is recorded as a function of the potential applied to the working electrode, and at any electrode potential the measured current is the sum of the capacitive current and the faradaic current. ${ }^{54}$ The capacitive current is originated from the structure of the electrode-solution interface, while the faradic current is measured when charges flow across the interface. Rectangular region of $\mathrm{CV}$ voltammograms are related to the capacitive current and slope regions are referred to the faradaic current. As can be seen in Fig. 4, a higher faradic current was achieved during electropolymerization of pyrrole in AN (IP-AN$\mathrm{CV}$ ), revealing a faster red/ox reactions across the electrodesolution interface, and so confirming a higher nucleation and polymerization rate. However, a slightly higher capacitive current was achieved in the case of electropolymerization in water (IP-W-CV) because of higher dielectric constant of water. The observed differences in the electropolymerization of PPy in AN and water (using both techniques) was in good agreement with the results obtained from SEM study of the samples, which would be discussed in next section.

\subsection{Surface morphology of actuators}

As discussed earlier, the magnitude and speed of the deformation of Nafion-based actuators depends on the structure and capacitance of the electrodes and also the nature of the interface between the electrode and the polymer. ${ }^{16} \mathrm{Fig} .5(\mathrm{a}-\mathrm{d})$ shows longitudinal SEM images of the electrode region of the actuators; (a) IP-W-CV, (b) IP-W-CHP, (c) IP-AN-CV, and (d) IP-ANCHP. The surface morphology is an important parameter as the PPy films are used in electromechanical applications. A uniform cauliflower-like deposition of PPy layer can be observed for all actuators. The functional groups on MWCNTs are likely to offer the interfacial interaction between the polymer and the nanotubes due to the hydrogen bonds formed between the MWCNTs and NH groups of the electrode layer. ${ }^{48}$ Comparison of the actuators in each row reveals the effect of the applied regime (using the same solvent) on the nanostructure of the PPy electrode region. As the images show, the surface morphology of the actuators changed considerably as a function of the applied regime. It is important to note that the size of the PPy grains was significantly reduced in actuators prepared using CHP technique, shown in Fig. 5(b) and (d). The largest PPy grains were formed in IP-W-CV actuator prepared in water using $\mathrm{CV}$ technique. Comparison of the actuators in each column also shows that the size of the PPy grains decreased considerably in actuators prepared in AN, shown in Fig. 5(c) and (d). The finest cauliflower-like PPy grains were formed in IP-AN-CHP actuator prepared in AN using CHP technique. This can be explained by the higher rate of nucleation and polymerization of pyrrole radical cations in AN (see Fig. 3). Fig. 6(a) and (b) shows cross sectional SEM images of the electrode region of the actuators; (a) IP-W-CHP and (b) IP-AN-CHP. The dark regions in these images show the Nafion membrane and the bright layer running along the surface of the membrane is assigned as the PPy-coated $\mathrm{CB} / \mathrm{MWCNT}$ electrode region. The surface layer in the IP-AN-CHP (Fig. 6(b)) actuator seems to be smoother and more uniform. The electrode layer appeared to have a higher thickness ( $\sim 2$ times) in the IP-AN-CHP actuators $(0.55 \mu \mathrm{m})$ compared to that in IP-W-CHP actuator $(0.25 \mu \mathrm{m})$. Electropolymerization of pyrrole in water can involve the side reaction of the cation radicals with water, yielding undesirable electrochemically inert products, ${ }^{50}$ resulting in the formation of shorter polymer chains with poorer adhesion properties compared with PPy film prepared in AN. 

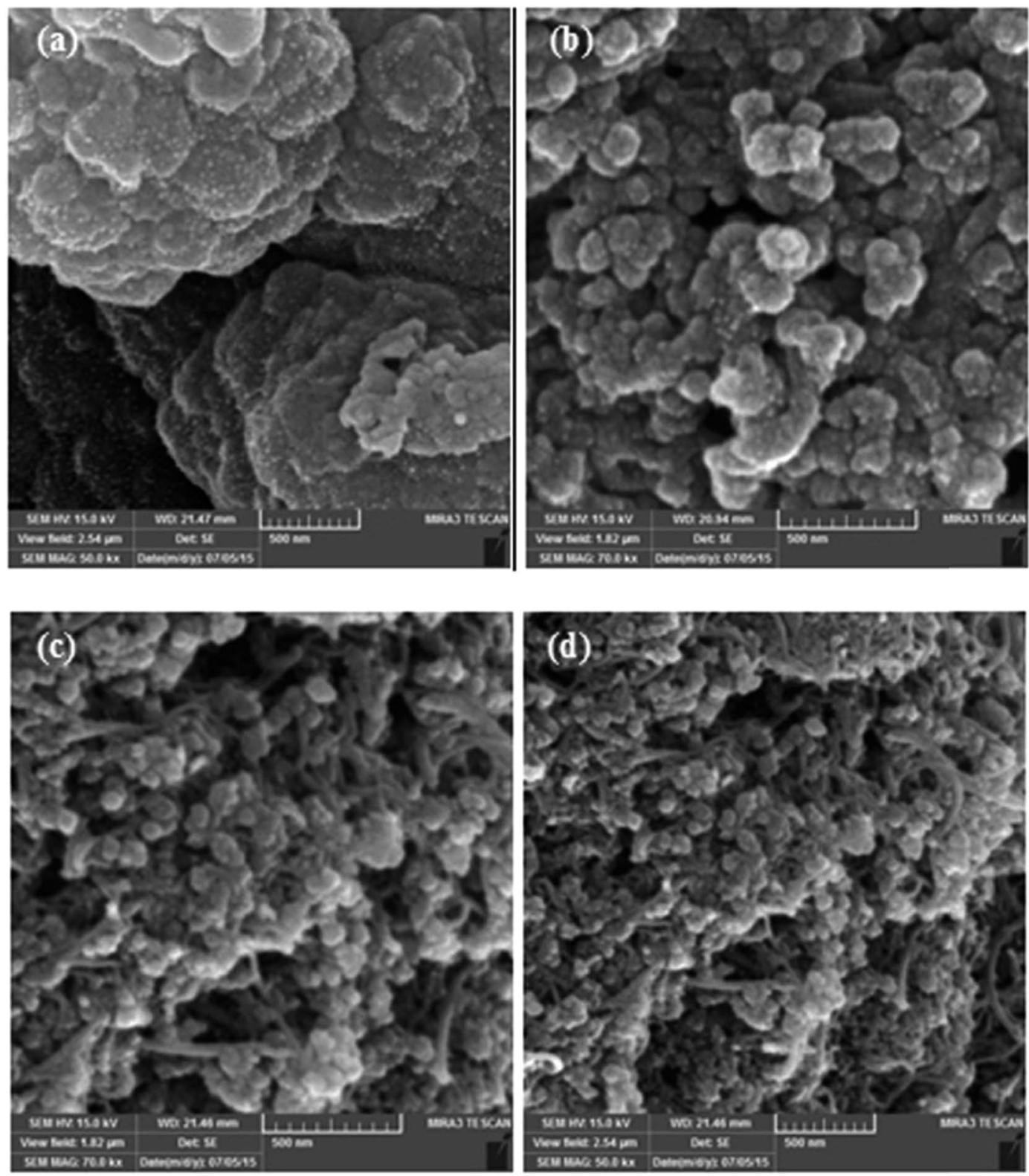

Fig. 5 Longitudinal SEM images of the electrode region of the actuators; (a) IP-W-CV, (b) IP-W-CHP, (c) IP-AN-CV, and (d) IP-AN-CHP.

\subsection{Physical characterization of actuators}

The data obtained from the evaluation of WUP and IEC of the prepared actuator (shown in Table 2) revealed that the metalfree actuators have higher water and ionic content compared to the Pt-based actuators. The hydrated thickness, WUP and IEC of IP-W-CV actuator, which appeared to have the largest PPy grains at the electrode region (see Fig. 5), was about 28\%, 60\% and $50 \%$ higher than that obtained for Pt-based IP actuator, respectively. These parameters increased significantly for the IP-W-CHP actuator, prepared in water using CHP technique, indicating the effect of the applied regime on the physical properties of the actuators. Replacing water with AN, resulted in a considerable increase in the hydrated thickness, WUP and IEC of the actuators, in which IP-AN-CHP showed the highest values. Furthermore, the IP-AN-CHP actuator showed to have higher IEC $(\sim 134 \%)$ and WUP $(\sim 53 \%)$ compared to that of the IP-W-CHP sample, indicating the effect of the applied solvent. The results demonstrate that the physical characteristics of the actuators change considerably as a function of the applied regime and the solvent. The variation in IEC and WUP of the actuators as a function of the applied synthesis parameters can be followed more clearly in Fig. 7. The observed changes could be attributed to the morphological differences of electrode layer in the fabricated actuators, as shown in Fig. 5. The electrode layer in the IP-AN-CHP actuators appeared to have the finest structure among all actuators. This facilitates the processes of water absorption and ion exchange in these actuators. It is interesting to note that the IP-AN-CHP actuator have WUP and IEC of about 3 and 6 times higher than that considered for Pt- 

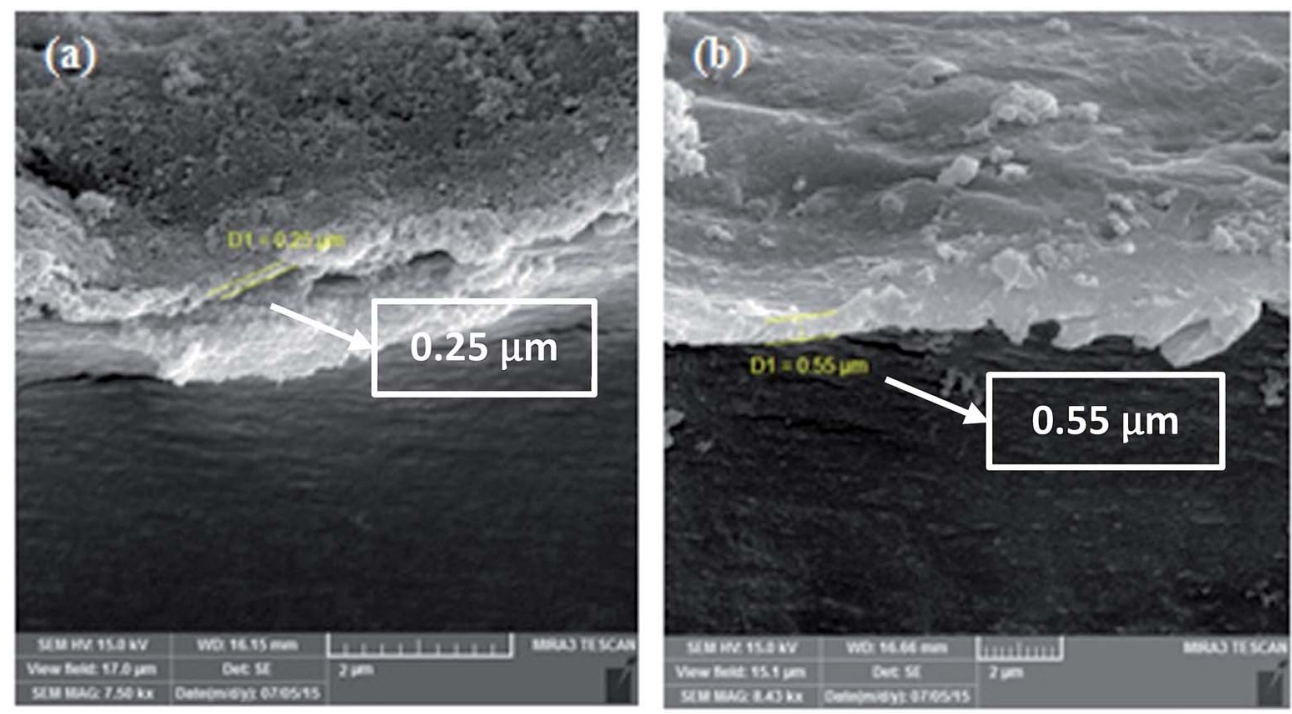

Fig. 6 Cross sectional SEM images of the electrode region of the actuators; (a) IP-W-CHP and (b) IP-AN-CHP.

Table 2 Hydrated thickness, ion exchange capacity (IEC) and water uptake (WUP) of actuators

\begin{tabular}{llll}
\hline $\begin{array}{l}\text { Sample } \\
\text { name }\end{array}$ & $\begin{array}{l}\text { Hydrated thickness } \\
(\mathrm{mm})\end{array}$ & $\begin{array}{l}\text { WUP } \\
(\%)\end{array}$ & $\begin{array}{l}\text { IEC } \\
\left(\mathrm{meq} \mathrm{g}^{-1}\right)\end{array}$ \\
\hline IP & 0.25 & 26 & 1.11 \\
IP-W-CV & 0.32 & 41.7 & 1.67 \\
IP-W-CHP & 0.36 & 52.0 & 2.85 \\
IP-AN-CV & 0.38 & 66.7 & 5.33 \\
IP-AN-CHP & 0.40 & 79.9 & 6.66
\end{tabular}

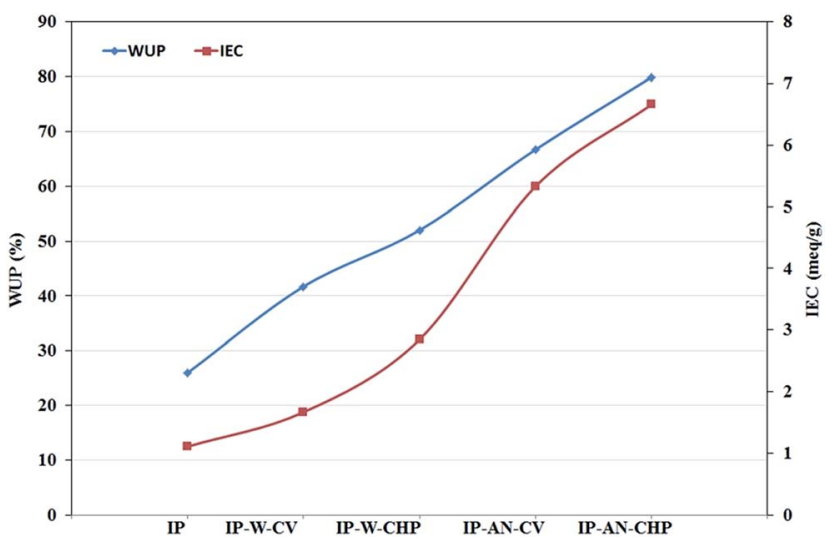

Fig. 7 Comparison of the IEC and WUP of the fabricated actuators.

electrode based IP actuator, respectively. This emphasize that the deposition of PPy film and the CB/MWCNT ink on the Nafion membrane not only led to no restriction in water absorption into the Nafion membrane but also it increased the water content of these actuators compared to Pt-based actuators. This can be attributed to the higher porosity and hydrophilicity of the electrode layer in IP-AN-CHP actuator compared to the metallic platinum electrode.

\subsection{Electrochemical characterizations of actuators}

The actuation performance of conventional Nafion-based actuators is directly related to the capacitance of the electrodes. The ability of electrodes to exhibit capacitive characteristic strongly depends on the pore structure and chemical affinity of the applied electrode material. As SEM images of the actuators (shown in Fig. 5 and 6) revealed the surface morphology and pore structure of the electrodeposited PPy film changed considerably by varying the synthesis parameters. Fig. 8 compares the cyclic voltammograms of the fabricated actuators to examine the effect of the electrode layer structure on the capacitive characteristic of the actuators. As can be seen, all CV voltammograms are approximately similar in shape but differ in the magnitude of the current. The symmetric shape of the CV curves revealed that the electropolymerization of PPy has given rise to two equivalent electrodes in all actuators, providing excellent charge distribution in the electrode regions. Pt-based IP actuator showed the narrowest CV loop and the current response increased in the order: IP-AN-CHP > IP-AN-CV

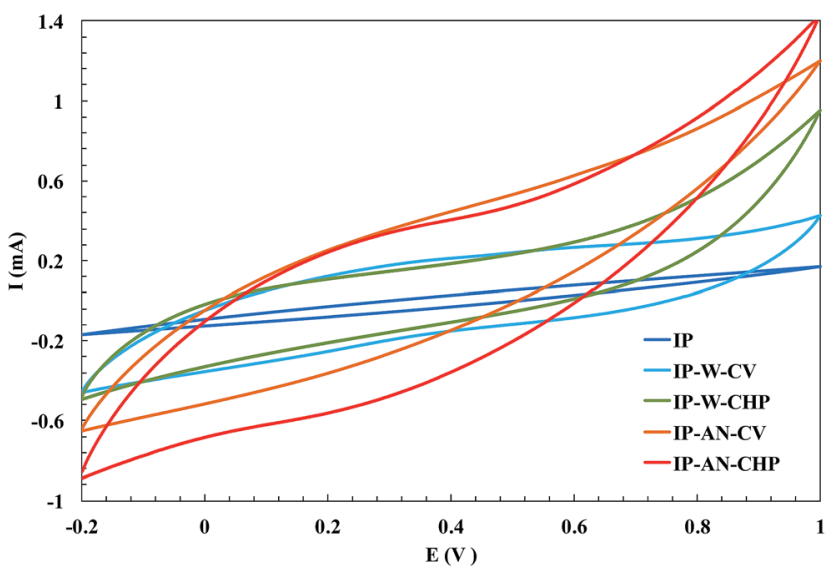

Fig. 8 CV response of the fabricated actuators. 

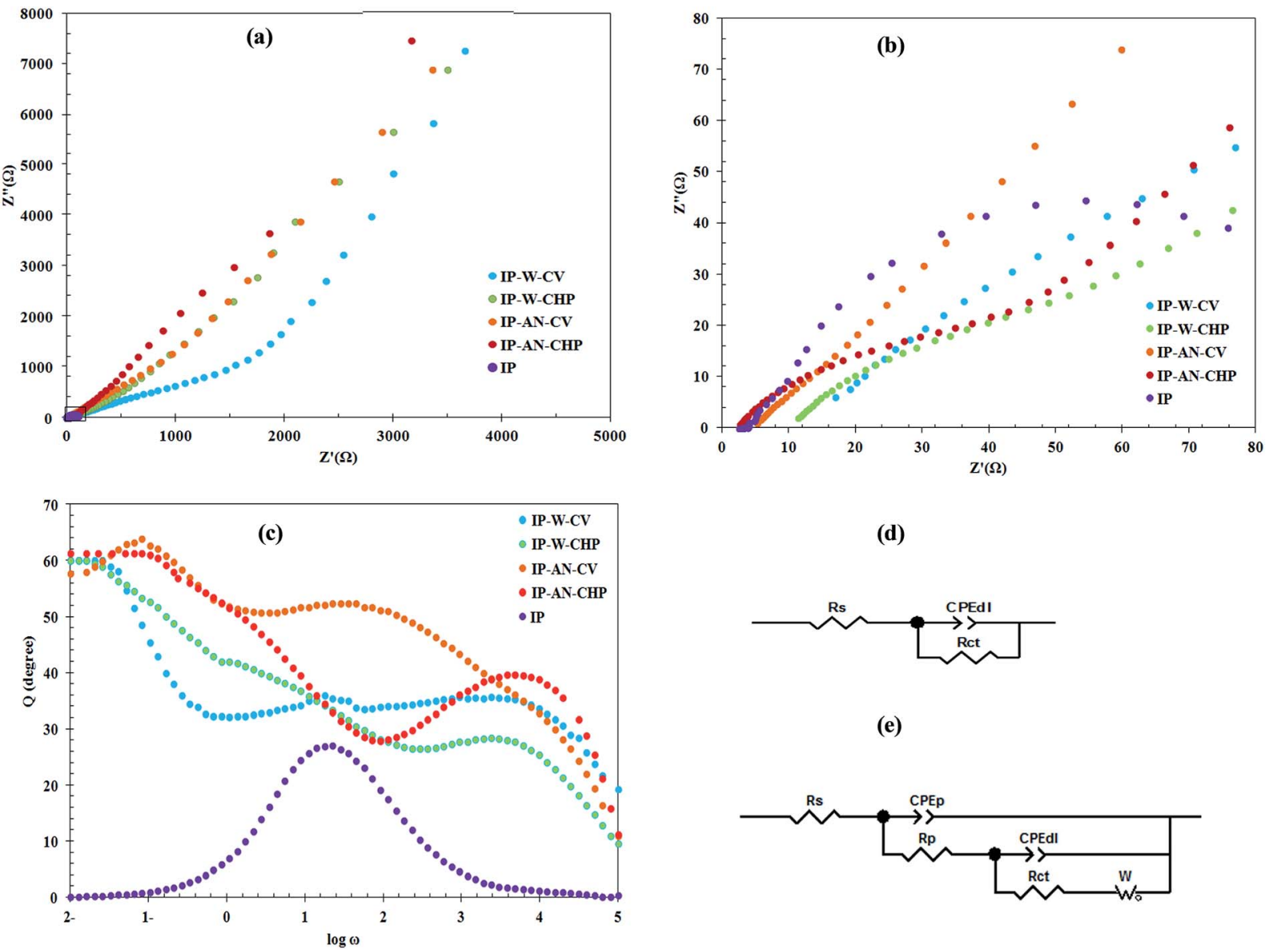

(d)

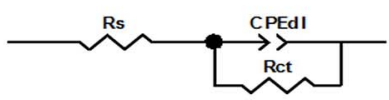

(e)

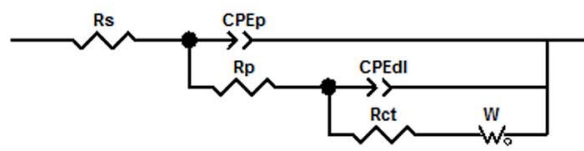

Fig. 9 Comparing Nyquist plots of actuators (a) in full view and (b) in the selected region shown in first part, (c) comparing Bode phase plots of actuators, (d) and (e) the proposed equivalent circuit model for Pt- and PPy/CB/MWCNT-electrode based actuators, respectively.

$>$ IP-W-CHP > IP-W-CV > IP. This indicates that the PPy/CB/ MWCNT-electrode based actuators generally show higher current response - and therefore higher capacitive characteristic. The results showed that the formation of the finest structure of electrode layer in the IP-AN-CHP actuator, which contributed in higher WUP and IEC of this actuator, also led to the highest current response. The more easily polarization mechanisms act, the larger current response and capacitive characteristic of the actuators would be. ${ }^{12}$ Therefore, it is expected that the polarization mechanisms happen easier in the IP-AN-CHP actuator since it has a more porous electrode structure which is more accessible to the solvated ions. The CV results were confirmed with the data obtained from EIS studies on the actuators.

Fig. 9(a-d) demonstrates the Nyquist plots, Bode phase plots and the equivalent circuits obtained by EIS for the fabricated actuators. As can be seen, Nyquist plots (shown in Fig. 9(a)) for all actuators are nearly linear in the low frequency region and have a semicircle shape in the high frequency region. Amongst all actuators, the steepest vertical line and the largest semicircle region was observed for IP-AN-CHP and IP-W-CV actuators, respectively. This suggests that the IP-AN-CHP actuators possess the highest charge storage capacity and the IP-W-CV actuators have the highest charge transfer resistance. For IPAN-CHP and IP-AN-CV actuators, the semicircle region appeared in higher frequency ranges compared to that of the IPW-CHP and IP-W-CV actuators, indicating lower ionic resistance of the actuators prepared in AN. This was attributed to the higher surface area of PPy film formed in AN and also to the higher accessibility of the ions to the more porous electrode structure in this actuator. The smallest semicircle was however obtained for Pt-based IP actuators at the far left of the $x$-axis, implying the lowest polarization resistance of IP among all actuators. Fig. 9(b) shows that the intercepts of the plots on the $x$-axis shifted to higher frequencies when water was replaced with AN, and CHP was used instead of CV technique. The high frequency intercepts on the real axis ( $x$-axis) were used to calculate the ionic resistance of the actuators. IP-AN-CHP actuators showed to have the lowest ionic resistance due to its higher IEC and WUP (see Table 2). EIS results also revealed that replacing water with AN in electropolymerization of PPy film has an enhancing impact on the capacitive characteristic and the ionic conduction of the actuators, particularly when CHP method is applied. Fig. 9(c) compares the Bode phase plots of EIS data to study the frequency response of actuators as a function of the electrode structure. Except for Pt-based IP 
Table 3 Equivalent circuit parameters of actuators obtained from EIS analysis

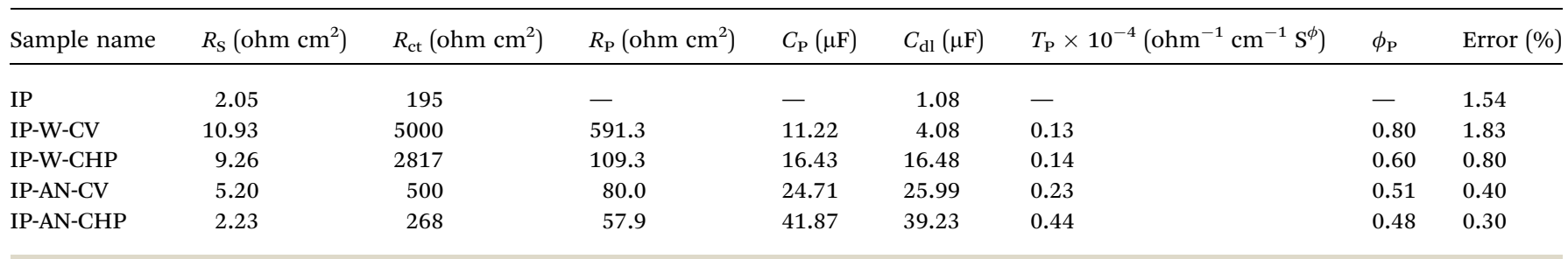

actuator, all Bode phase plots are approximately similar in shape and exhibit two peaks, which were assigned as two time constants. The intercept of the plots on the $y$-axis was used to recognize the electrochemical behavior of the actuators in the low frequencies. PPy-based actuators showed a phase angle in the range of $59^{\circ}$ to $61^{\circ}$, indicating the diffusion-limited mass transport of these actuators at low frequency regions. ${ }^{55}$ The impedance data was then fit with an equivalent circuit model to estimate contributions of the electrode components to the overall electrical properties. The chosen equivalent electrical circuit model for IP and PPy-based actuators are depicted in Fig. 9(d) and (e), respectively. The Randles model (shown in Fig. 9(d)) was found to give excellent fit for EIS data of IP actuators. The chosen model presented for PPy-based actuators composed of $R_{\mathrm{S}}, \mathrm{CPE}_{\mathrm{P}}, R_{\mathrm{P}}, \mathrm{CPE}_{\mathrm{dl}}, R_{\mathrm{ct}}$ and $W$ elements that represent the solution resistance, pseudo-capacitance, charge transfer resistance of the polymer, double layer capacitance, charge transfer resistance of double layer and Warburg diffusion element of the actuators, respectively. The $\mathrm{CPE}_{\mathrm{dl}} \| R_{\mathrm{ct}}$ component is originated from the charging-discharging process at the electrolyte/electrode interface. The $\mathrm{CPE}_{\mathrm{P}} \| R_{\mathrm{P}}$ component is arisen from the presence of counter-anions besides the available positive charges in the electrode layer. $\mathrm{CPE}$ is non-ideal capacitor which its impedance can be calculated according to eqn (3): $:^{31,56}$

$$
Z_{\mathrm{CPE}}=1 / T(\mathrm{j} \omega)^{\phi}
$$

where $T$ and $\phi$ are the parameters that are independent of the frequency. $T$ is the amplitude comparable to a capacitance (admittance) and the exponent, $\phi$, shows the roughness of the electrode with values from 0 to 1 . An ideal CPE shows $\phi=1$, while $\phi=0$ shows resistance and for the Warburg diffusion behavior $\phi=0.5{ }^{.77}$ The parameters of the equivalent circuit for all actuators are shown in Table 3. The mean error for all actuators was less than $2 \%$, while the lowest mean error was obtained for the IP-AN-CHP actuator $(0.3 \%)$, indicating the perfect fitting of the proposed equivalent circuit model to the EIS data of the PPy-based actuators. IP-W-CV actuators showed the highest $\phi$, indicating the lowest surface roughness (porosity) of the electrode layer in this actuator. The $\phi$ decreased in the order: IP-W-CV > IP-W-CHP > IP-AN-CV > IP-AN-CHP. The lowest $\phi_{\mathrm{p}}$ belonged to the IP-AN-CHP actuator with the finest electrode structure and the highest surface porosity. The observed trend for $\phi$ was reversed for the calculated $T$ in which the IP-AN-CHP actuator showed the highest $T$, due to its highest capacitive characteristic. For actuators, the true capacitance $(C)$ was calculated by the following equation: ${ }^{58,59}$

$$
C=\left[T /\left(R_{\mathrm{S}}^{-1}+R_{\mathrm{ct}}^{-1}\right)^{(1-\phi)}\right]^{1 / \phi}
$$

The variation in the double layer capacitance $\left(C_{\mathrm{dl}}\right)$, pseudo capacitance $\left(C_{\mathrm{P}}\right)$ and ionic conductivity $(\sigma)$ of the prepared actuators can be followed more clearly in Fig. 10. As can be seen, the IP-AN-CHP actuator exhibited the highest $C_{\mathrm{P}}, C_{\mathrm{dl}}$ and $\sigma$ values. This was attributed to the high surface porosity of the electrode region and high ionic and water content of this actuator. In contrast, the IP-W-CV actuator showed the highest $R_{\mathrm{ct}}$ and $R_{\mathrm{P}}$ values due to the formation of large PPy grains with lower surface area at both faces of Nafion membrane. The results also revealed that the IP-AN-CHP actuator possessed $\sigma$, $C_{\mathrm{dl}}$ and $C_{\mathrm{P}}$ of about twenty three, ten and four times higher than that of IP-W-CV actuators, respectively. Increasing the $C_{\mathrm{P}}$ in these actuators can be explained by $C_{\mathrm{P}}=A \varepsilon / d$, where $\varepsilon, A$ and $d$ are the permittivity coefficient of the Nafion membrane, the surface area of the electrode and distance between the electrodes (the thickness of Nafion), respectively. ${ }^{56}$ The values of $\varepsilon$ and $d$ can be considered approximately constant for all actuators. The SEM images (Fig. 5 and 6) showed that the surface morphology of the actuators changed considerably by altering the synthesis parameters. Thus, $C_{\mathrm{P}}$ of actuators can be directly related to the surface area of the electrode region. The observed higher $C_{\mathrm{P}}$ of IP-AN-CHP actuators can be attributed to their finest electrode structure, as shown in Fig. 5. This actuator also

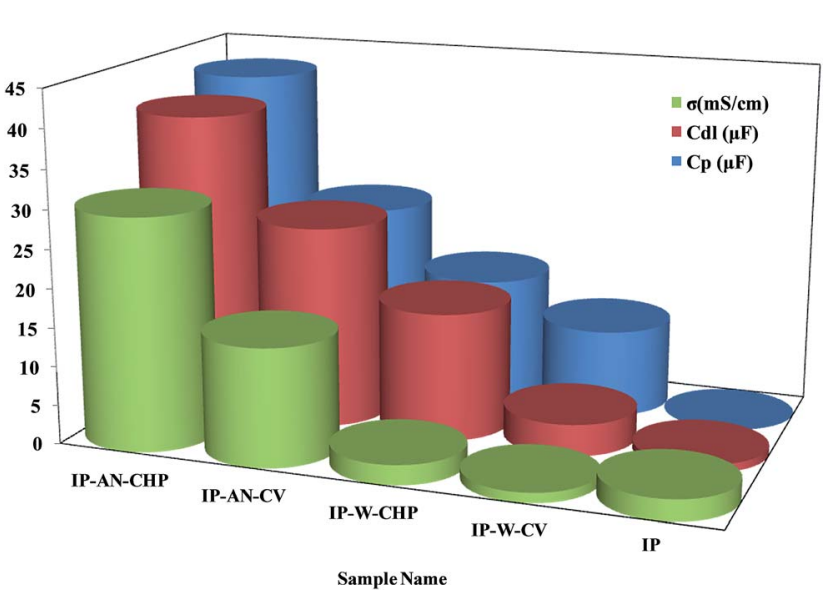

Fig. 10 The variation in the double layer capacitance $\left(C_{\mathrm{dl}}\right)$, pseudo capacitance $\left(C_{P}\right)$ and ionic conductivity $(\sigma)$ of all actuators. 


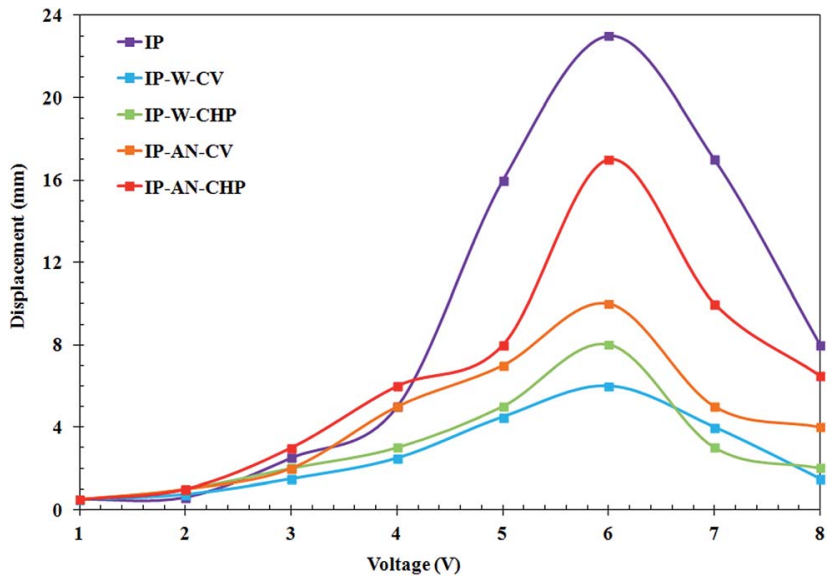

Fig. 11 Comparison of the dependence of tip displacement on amplitude of the input d.c. voltage for the fabricated actuators.

showed to have $C_{\mathrm{dl}}$ and $\sigma$ of about thirty six and eleven times higher that considered for Pt-based IP actuator, respectively, while $R_{\text {ct }}$ of the IP-AN-CHP actuator was about $37 \%$ higher. This suggests that the more porous and flexible structure of the electrode layers in the PPy-based actuator contributed in the formation of larger double layers, accommodating a higher amount of electrolyte and facilitating the insertion and extraction of ions at the electrode regions. It has been established there are some associations among the quinoid rings of PPy and MWCNTs which facilitate the charge transfer between the two components; PPy behaves as a good electron donor while MWCNTs act as good electron acceptors. ${ }^{60}$ Thus, it is expected that the surface conductivity of the PPy-electrode based actuators can be improved by varying the synthesis parameters to provide a stronger coupling between the MWCNTs and the PPy chains.

\subsection{Actuation performance of actuators}

The average maximum tip displacement of the free end of the fabricated actuators was measured as a function of the amplitude of the applied d.c. voltage. Fig. 11 demonstrates that the tip displacement of all actuators increased as a function of input voltage in potential range of 1 to $6 \mathrm{~V}$, and afterward decreased by

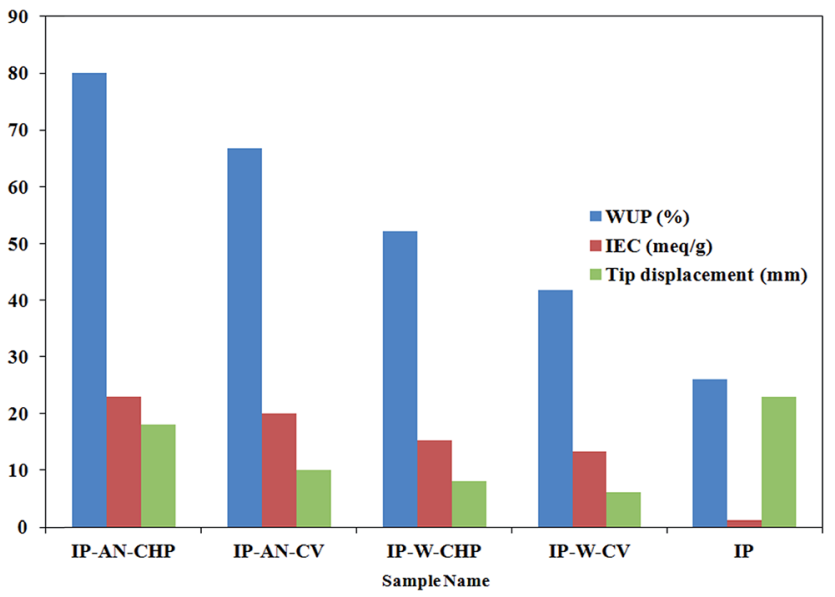

Fig. 13 The average maximum tip displacement, IEC $\left(\right.$ meq $\mathrm{g}^{-1}$ ) and WUP (\%) of all actuators, in response to the same d.c. voltage of $6 \mathrm{~V}$ in amplitude.

applying higher voltages. The comparison of the tip displacement of actuators also revealed that the magnitude of tip displacements were dependent on the applied synthesis parameters for the electropolymerization of the PPy film. The corresponding overlaid digital images captured at the starting point and end point for actuators (a) IP-W-CV, (b) IP-W-CHP, (c) IP-AN-CV and (d) IP-AN-CHP (with the corresponding SEM images) in response to d.c. voltage of $6 \mathrm{~V}$ in amplitude are shown in Fig. 12. The maximum tip displacement of actuators followed the order of IP-AN-CHP $>$ IP-AN-CV $>$ IP-W-CHP $>$ IP-W$\mathrm{CV}$. The IP-AN-CHP actuator which showed to have the highest WUP, IEC, ionic conductivity and electrode capacitance presented the highest tip displacements $(\sim 17 \mathrm{~mm})$ under $6 \mathrm{~V}$ d.c. potential which was about two and three order of magnitude higher than that considered for IP-AN-CV $(\sim 10 \mathrm{~mm})$ and IP-W$\mathrm{CV}(\sim 6 \mathrm{~mm})$ actuators. The variation in the WUP, IEC and the maximum tip displacement of actuators can be followed in Fig. 13. It is important to note that the IP-AN-CHP actuator with the highest hydrated thickness showed the highest tip displacement. This shows that the deposition of a thicker PPy film (see Fig. 6) promotes higher water absorption while it does not restrict the bending deformation of the actuator. The
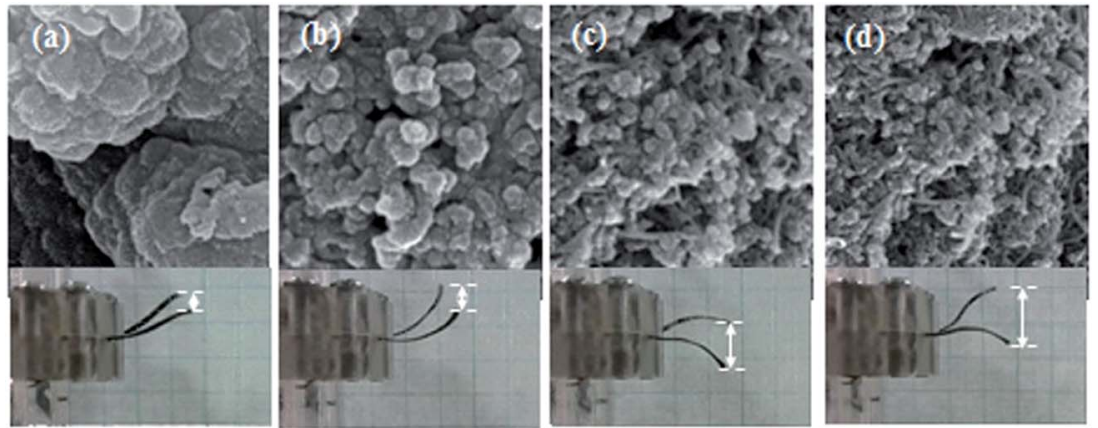

Fig. 12 The corresponding overlaid digital images captured at the starting point and end point for actuators (a) IP-W-CV, (b) IP-W-CHP, (c) IPAN-CV and (d) IP-AN-CHP (with their corresponding SEM images) in response to d.c. voltage of $6 \mathrm{~V}$ in amplitude. 

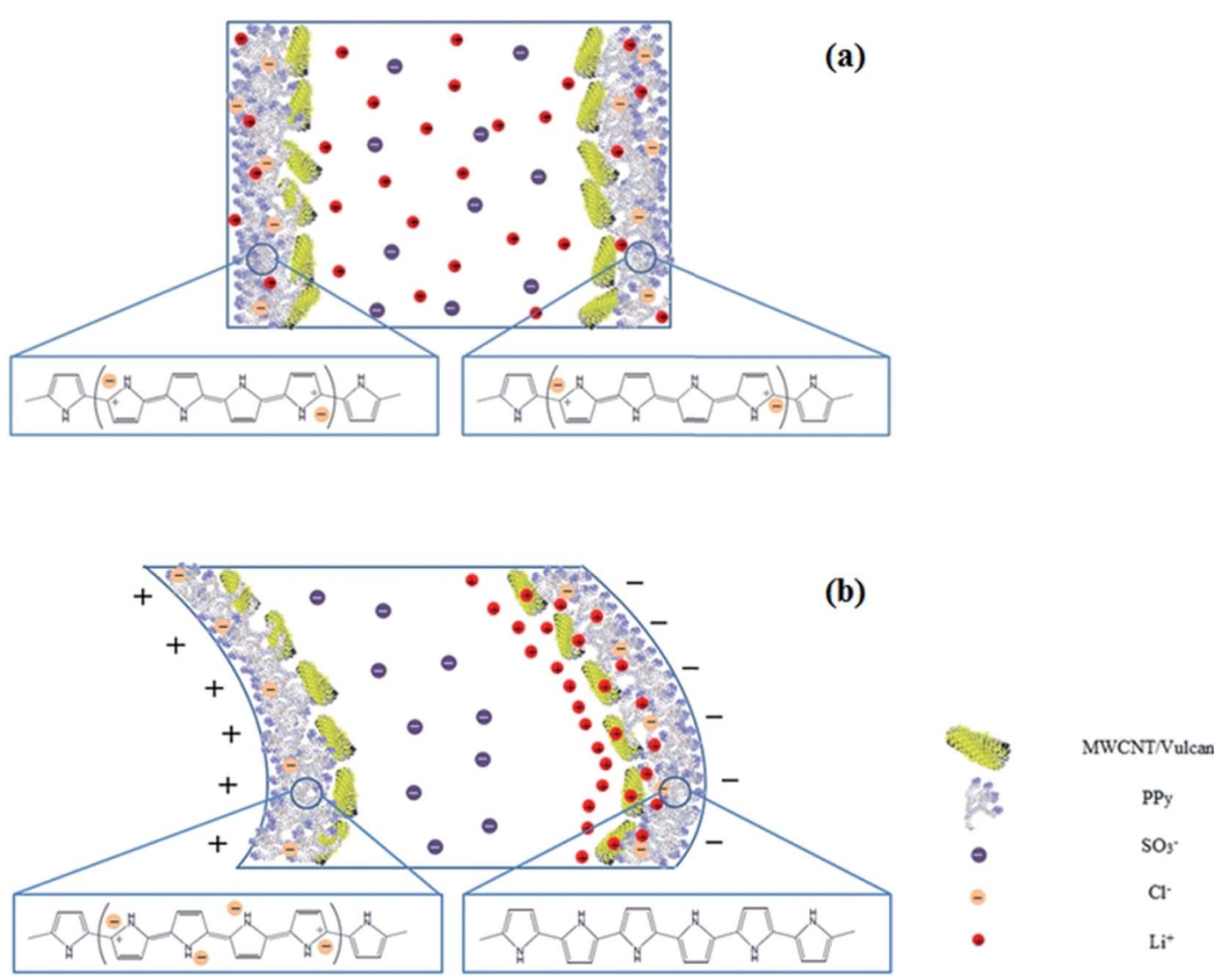

Fig. 14 Schematic representation of ion-redistribution in PPy-electrode actuators (a) with no potential applied and (b) in response to a d.c. voltage.

maximum tip displacement of this actuator was however about $26 \%$ lower than that of the conventional Pt electrode-based IPMC actuators fabricated in previous studies ${ }^{\mathbf{1 1}}$ which can be improved by decreasing surface resistance of the electrode layer by changing synthesis parameters of PPy film. In addition, it is expected that the IP-AN-CHP actuator to show a higher durability (life time) compared to the conventional IPMC actuators since it can absorb water in significantly higher extent $(\sim 3$ times). Fig. 14 illustrates a schematic depiction of the redistribution of ions under influence of applied electric field and formation of electric double layer at the interface of Nafion and $\mathrm{PPy} / \mathrm{CB} / \mathrm{MWCNT}$ electrode. As Fig. 14(a) demonstrates when no potential is applied $\mathrm{Li}^{+}$ions are mainly located within the Nafion membrane while a smaller portion of these ions are trapped in porous structure of the electrode layer at both sides. On application of an electric voltage, $V$, opposite charges of equal magnitude, $Q$, accumulate on the electrodes. The charge can be calculated according to eqn (5), where $C$ is the capacitance of the actuators. ${ }^{7}$

$$
Q=C V
$$

Accumulation of opposite charges on the electrodes produces an electric field gradient developing across the thickness of the actuators. It is expected that the PPy chains become reduced at the negatively charged electrode (cathode), shown in Fig. 14(b). In this case, the hydrated $\mathrm{Li}^{+}$ions begin to break away from the sulfonate groups within Nafion membrane and move towards the cathode, while the sulfonate groups remain fixed to the molecular backbone. Since Nafion only pass cations, the chloride ions cannot diffuse electrically towards the anode and remain in the electrode region. Moreover, it has been established that MWCNTs change dimensions upon applying an external potential. ${ }^{61}$ The predominant actuation mechanism for the MWCNTs is the electrostatic repulsion between CNTs that are equally charged and the establishment of an electrostatic double layer which prompts the CNT layer to swell. ${ }^{61}$ This results in the formation of a thicker double layer near the cathode and bending deformation of the sample towards the anode. On application of a higher voltage to actuators with higher capacitance, more charge accumulates on the electrodes and a greater number of hydrated ions diffuse electrically towards the cathode. This produces a larger tip displacement in actuators with the highest capacitive characteristic (according to $\mathrm{CV}$ and EIS data, IP-AN-CHP actuator).

$\mathrm{CNTs}^{20,62}$ and $\mathrm{CPs}^{26}$ have been proposed as alternative electrode materials for the fabrication of ionic actuators. The work presented by Lee et $a .^{63}$ is a procedure for the fabrication of IPMC actuators with electrospun Nafion/MWCNT composite electrodes, in which the surfaces of composite electrodes were 
ion-beam coated with a thin gold layers to reduce the surface resistance. The proposed ionic actuator showed a rapid and large bending motion. They showed that the energy efficiency of strain was over 10 times higher than that of the conventional IPMC actuators. ${ }^{63}$ Various efforts have also been made to incorporate PPy into the Nafion membrane ${ }^{\mathbf{6 4 , 6 5}}$ in order to improve the performance of IPMC actuators. Although there are reports on the actuation performance of CP-based actuators ${ }^{34}$ the PPy-electrode Nafion-based actuators have not been reported previously. The attractive points of PPy includes low operating voltage, large electrochemical strain, and long cycle life $^{66}$ Furthermore, PPy film is non-toxic, lightweight, flexible, biocompatible and can provide sufficient surface conductivity and prevent dehydration of actuator. These are the requirements for a suitable electrode material for ionic actuators. As mentioned earlier, the magnitude and speed of the bending deformation of water-based actuators depends strongly on the IEC, WUP and $\sigma$ of the actuator. Compared to the conventional Pt-based IPMC actuator, ${ }^{7}$ the current work proposes a simple method for manufacturing high-performance ionic actuators using PPy-CB/MWCNT-coated Nafion membrane with improved hydrophilic, IEC, $\sigma$ and $C_{\mathrm{dl}}$ characteristic. This paper also provides new insights in the relationship between the $\mathrm{CB} /$ MWCNT/PPy electrode nanostructure and the synthesis parameters of PPy film and their effect on the actuation performance of the metal-free ionic actuators.

\section{Conclusion}

We have demonstrated for the first time the fabrication and characterization of $\mathrm{PPy} / \mathrm{CB} / \mathrm{MWCNT}$-electrode Nafion-based actuators. PPy film was electro-polymerized on the $\mathrm{CB} /$ MWCNT-coated Nafion membrane using CHP and CV techniques. The effect of solvent type and applied regime on the PPy film nanostructure and also the actuation performance of the actuators were followed using physical, electrochemical and electromechanical experiments and were compared with the corresponding behavior of Pt electrode-based IPMC actuators. WUP and IEC of actuators were determined and the impact of the applied technique and the solvent type on the morphology of PPy-based electrode layer was studied using SEM. Furthermore, $\mathrm{CV}$ responses, EIS data and actuation performance of actuators were compared to follow the effect of varying synthesis parameters on $R_{\mathrm{ct}}, C_{\mathrm{dl}}$ and $\sigma$ of actuators. Generally, actuators prepared using CHP technique in AN as solvent showed the highest hydrated thickness, IEC and WUP, which was about $3 \%, 17 \%$ and $6 \%$ higher than that considered for conventional Pt-based actuators, respectively. SEM analysis showed that the electrodeposition of PPy on CB/MWCNT-coated Nafion membrane resulted in the formation of PPy film with good surface coverage. The surface morphology of the electrode layer was found to be dependent on the solvent type and the applied regime. The CHP technique gave rise to finer and denser PPy grains in actuators prepared in AN. Furthermore, the electrode layer appeared to have higher thickness ( $\sim 2$ times) in these actuators compared to the polymer film electrodeposited in water using CHP technique. CV studies of actuators revealed that applying PPy film on CB/MWCNT-coated Nafion membrane generally led to a higher current response and therefore higher characteristic capacitance compared to the conventional Pt-based IPMC actuators. The actuators prepared using CHP technique in AN showed the highest $C_{\mathrm{dl}}$ and $\sigma$, and also the lowest $R_{\mathrm{ct}}$ among all actuators. Moreover, these actuators showed the highest ionic conductivity which was about twenty three and eleven times higher than that considered for actuators prepared using CV technique in water and Pt-based IPMC actuators, respectively. In electromechanical tests the maximum tip displacement were recorded for actuators. For all actuators, displacement increased by increasing applied voltage. The PPy-electrode actuators prepared using CHP technique in AN showed the highest tip displacement in all applied voltages. Moreover, the tip displacement of these actuators appeared to be about two times higher than the actuators prepared in water using $\mathrm{CV}$ technique. The maximum tip displacement of these actuators was however about $26 \%$ lower than that of the conventional Pt electrode-based IPMC actuators which could be improved by decreasing surface resistance of the PPy-based electrode layer by changing synthesis parameters.

\section{Acknowledgements}

Authors gratefully acknowledge Nanotechnology Initiative Council, Amirkabir University of Technology and Tabriz University for all funding supports through this project.

\section{References}

1 S. Mohsen and J. K. Kwang, Ionic polymer-metal composites: I. Fundamentals, Smart Mater. Struct., 2001, 10, 819.

2 Y. Bar-Cohen, Electroactive Polymer (EAP) Actuators as Artificial Muscles: Reality, Potential, and Challenges, SPIE Press, Washington, 2004.

3 K. Asaka and K. Oguro, IPMC Actuators: Fundamentals, Biomedical Applications of Electroactive Polymer Actuators, John Wiley \& Sons, Ltd, 2009, pp. 101-119.

4 K. Byungkyu, K. Deok-Ho, J. Jaehoon and P. Jong-Oh, A biomimetic undulatory tadpole robot using ionic polymermetal composite actuators, Smart Mater. Struct., 2005, 14, 1579.

5 M. Shahinpoor, K. J. Kim and M. Mojarrad, Artificial Muscles: Applications of Advanced Polymeric Nanocomposites, CRC Press, 2007.

$6 \mathrm{M}$. Shahinpoor and J. K. Kwang, Ionic polymer-metal composites: III. Modeling and simulation as biomimetic sensors, actuators, transducers, and artificial muscles, Smart Mater. Struct., 2004, 13, 1362.

7 L. Naji, J. A. Chudek, E. W. Abel and R. T. Baker, Electromechanical behaviour of Nafion-based soft actuators, J. Mater. Chem. B, 2013, 1, 2502-2514.

$8 \mathrm{H}$. Tamagawa and F. Nogata, Bending response of dehydrated ion exchange polymer membranes to the applied voltage, J. Membr. Sci., 2004, 243, 229-234. 
9 J. Lin, Y. Liu and Q. M. Zhang, Charge dynamics and bending actuation in Aquivion membrane swelled with ionic liquids, Polymer, 2011, 52, 540-546.

10 Y. Liu, C. Lu, S. Twigg, M. Ghaffari, J. Lin, N. Winograd and Q. M. Zhang, Direct Observation of Ion Distributions near Electrodes in Ionic Polymer Actuators Containing Ionic Liquids, Sci. Rep., 2013, 3, 973.

11 J. H. Park, M. J. Han, D. S. Song and J. Y. Jho, Ionic PolymerMetal Composite Actuators Obtained from RadiationGrafted Cation- and Anion-Exchange Membranes, ACS Appl. Mater. Interfaces, 2014, 6, 22847-22854.

12 M. Safari, L. Naji, R. T. Baker and F. A. Taromi, The enhancement effect of lithium ions on actuation performance of ionic liquid-based IPMC soft actuators, Polymer, 2015, 76, 140-149.

13 S. Nemat-Nasser, S. Zamani and Y. Tor, Effect of solvents on the chemical and physical properties of ionic polymer-metal composites, J. Appl. Phys., 2006, 99, 104902.

14 M. Aureli, W. Lin and M. Porfiri, On the capacitance-boost of ionic polymer metal composites due to electroless plating: theory and experiments, J. Appl. Phys., 2009, 105, 104911.

15 L. Naji, J. A. Chudek and R. T. Baker, Magnetic resonance imaging study of a soft actuator element during operation, Soft Matter, 2008, 4, 1879-1886.

16 L. Naji, M. Safari and S. Moaven, Fabrication of SGO/Nafionbased IPMC soft actuators with sea anemone-like Pt electrodes and enhanced actuation performance, Carbon, 2016, 100, 243-257.

17 Z. Xue, Y. Tang, X. Duan, Y. Ye, X. Xie and X. Zhou, Ionic polymer-metal composite actuators obtained from sulfonated poly(ether ether sulfone) ion-exchange membranes, Composites, Part A, 2016, 81, 13-21.

18 Y. Tang, Z. Xue, X. Zhou, X. Xie and C.-Y. Tang, Novel sulfonated polysulfone ion exchange membranes for ionic polymer-metal composite actuators, Sens. Actuators, B, 2014, 202, 1164-1174.

19 J. Kim, J.-H. Jeon, H.-J. Kim, H. Lim and I.-K. Oh, Durable and Water-Floatable Ionic Polymer Actuator with Hydrophobic and Asymmetrically Laser-Scribed Reduced Graphene Oxide Paper Electrodes, ACS Nano, 2014, 8, 2986-2997.

20 L. Kong and W. Chen, Carbon Nanotube and Graphenebased Bioinspired Electrochemical Actuators, Adv. Mater., 2014, 26, 1025-1043.

21 A. G. Pandolfo and A. F. Hollenkamp, Carbon properties and their role in supercapacitors, J. Power Sources, 2006, 157, 11-27.

22 G. W. Rogers and J. Z. Liu, High-Performance Graphene Oxide Electromechanical Actuators, J. Am. Chem. Soc., 2012, 134, 1250-1255.

23 N. Terasawa, N. Ono, Y. Hayakawa, K. Mukai, T. Koga, N. Higashi and K. Asaka, Effect of hexafluoropropylene on the performance of poly(vinylidene fluoride) polymer actuators based on single-walled carbon nanotube-ionic liquid gel, Sens. Actuators, B, 2011, 160, 161-167.

24 C. Li, D. Wang, T. Liang, X. Wang and L. Ji, A study of activated carbon nanotubes as double-layer capacitors electrode materials, Mater. Lett., 2004, 58, 3774-3777.
25 N. Terasawa and K. Asaka, High-Performance PEDOT:PSS/ Single-Walled Carbon Nanotube/Ionic Liquid Actuators Combining Electrostatic Double-Layer and Faradaic Capacitors, Langmuir, 2016, 32, 7210-7218.

26 K. Haenen, J. A. Garrido, J. Robertson, N. Terasawa and K. Asaka, Electrochemical and Electromechanical Properties of Activated Multi-walled Carbon Nanotube Polymer Actuator that Surpass the Performance of a Singlewalled Carbon Nanotube Polymer Actuator, Materials Today: Proceedings, 2016, 3, S178-S183.

27 N. Terasawa, K. Mukai, K. Yamato and K. Asaka, Superior performance of manganese oxide/multi-walled carbon nanotubes polymer actuator over ruthenium oxide/multiwalled carbon nanotubes and single-walled carbon nanotubes, Sens. Actuators, B, 2012, 171-172, 595-601.

28 J. Torop, V. Palmre, M. Arulepp, T. Sugino, K. Asaka and A. Aabloo, Flexible supercapacitor-like actuator with carbide-derived carbon electrodes, Carbon, 2011, 49, 31133119.

29 J. Torop, T. Sugino, K. Asaka, A. Jänes, E. Lust and A. Aabloo, Nanoporous carbide-derived carbon based actuators modified with gold foil: prospect for fast response and low voltage applications, Sens. Actuators, B, 2012, 161, 629-634.

30 M. G. Hosseini and P. Zardari, Electrocatalytical study of carbon supported $\mathrm{Pt}, \mathrm{Ru}$ and bimetallic Pt-Ru nanoparticles for oxygen reduction reaction in alkaline media, Appl. Surf. Sci., 2015, 345, 223-231.

31 M. Hosseini, E. Shahryari, R. Najjar and I. Ahadzadeh, Study of Super Capacitive Behavior of Polyaniline/manganese Oxide-Carbon Black Nanocomposites Based Electrodes, Int. J. Nanosci. Nanotechnol., 2015, 11, 147-157.

32 R. Balint, N. J. Cassidy and S. H. Cartmell, Conductive polymers: towards a smart biomaterial for tissue engineering, Acta Biomater., 2014, 10, 2341-2353.

33 A. Khaldi, D. Falk, A. Maziz and E. W. H. Jager, Fabrication and adhesion of conjugated polymer trilayer structures for soft, flexible micromanipulators, 2016, p. 97980N-97988.

34 Q. Liu, L. Liu, K. Xie, Y. Meng, H. Wu, G. Wang, Z. Dai, Z. Wei and Z. Zhang, Synergistic effect of a r-GO/PANI nanocomposite electrode based air working ionic actuator with a large actuation stroke and long-term durability, $J$. Mater. Chem. A, 2015, 3, 8380-8388.

35 J. G. Martinez and T. F. Otero, Structural electrochemistry. Chronopotentiometric responses from rising compacted polypyrrole electrodes: experiments and model, RSC Adv., 2014, 4, 29139-29145.

36 N. Terasawa and I. Takeuchi, Electrochemical and electromechanical properties of carbon black/carbon fiber composite polymer actuator with higher performance than single-walled carbon nanotube polymer actuator, Electrochim. Acta, 2014, 123, 340-345.

37 F. Vidal, J. F. Popp, C. Plesse, C. Chevrot and D. Teyssié, Feasibility of conducting semi-interpenetrating networks based on a poly(ethylene oxide) network and poly(3,4ethylenedioxythiophene) in actuator design, J. Appl. Polym. Sci., 2003, 90, 3569-3577. 
38 M. S. Ramasamy, S. S. Mahapatra, H. J. Yoo, Y. A. Kim and J. W. Cho, Soluble conducting polymer-functionalized graphene oxide for air-operable actuator fabrication, $J$. Mater. Chem. A, 2014, 2, 4788-4794.

39 G. Wu, Y. Hu, Y. Liu, J. Zhao, X. Chen, V. Whoehling, C. Plesse, G. T. M. Nguyen, F. Vidal and W. Chen, Graphitic carbon nitride nanosheet electrode-based highperformance ionic actuator, Nat. Commun., 2015, 6, 7258.

40 Z. Zeng, H. Jin, L. Zhang, H. Zhang, Z. Chen, F. Gao and Z. Zhang, Low-voltage and high-performance electrothermal actuator based on multi-walled carbon nanotube/polymer composites, Carbon, 2015, 84, 327-334.

41 T. May, T. Van-Tan, M. S. Geoffrey and G. W. Gordon, Carbon nanotube and polyaniline composite actuators, Smart Mater. Struct., 2003, 12, 626.

42 C. Peng, S. Zhang, D. Jewell and G. Z. Chen, Carbon nanotube and conducting polymer composites for supercapacitors, Prog. Nat. Sci., 2008, 18, 777-788.

43 D. Salinas-Torres, J. M. Sieben, D. Lozano-Castelló, D. Cazorla-Amorós and E. Morallón, Asymmetric hybrid capacitors based on activated carbon and activated carbon fibre-PANI electrodes, Electrochim. Acta, 2013, 89, 326-333.

44 P. Saville, Polypyrrole Formation and Use, Defence R\&D Canada - Atlantic Technical Memorandum DRDC Atlantic TM 2005-004, Jan 2005.

45 D. Sangian, W. Zheng and G. M. Spinks, Optimization of the sequential polymerization synthesis method for polypyrrole films, Synth. Met., 2014, 189, 53-56.

46 J. Torop, A. Aabloo and E. W. H. Jager, Novel actuators based on polypyrrole/carbide-derived carbon hybrid materials, Carbon, 2014, 80, 387-395.

47 S. Chen and I. Zhitomirsky, Capacitive behaviour of polypyrrole, prepared by electrochemical and chemical methods, Mater. Lett., 2014, 125, 92-95.

48 B. Zhang, Y. Xu, Y. Zheng, L. Dai, M. Zhang, J. Yang, Y. Chen, X. Chen and J. Zhou, A Facile Synthesis of Polypyrrole/ Carbon Nanotube Composites with Ultrathin, Uniform and Thickness-Tunable Polypyrrole Shells, Nanoscale Res. Lett., 2011, 6, 431.

49 L. Naji, J. A. Chudek and R. T. Baker, Time-Resolved Mapping of Water Diffusion Coefficients in a Working Soft Actuator Device, J. Phys. Chem. B, 2008, 112, 9761-9768.

50 V. V. Tat'yana and N. E. Oleg, Polypyrrole: a conducting polymer; its synthesis, properties and applications, Russ. Chem. Rev., 1997, 66, 443.

51 H. Shokry Hassan, A. B. Kashyout, I. Morsi, A. A. A. Nasser and H. Abuklill, Development of polypyrrole coated copper nanowires for gas sensor application, Sens. Biosensing Res., 2015, 5, 50-54.

52 M. J. Martínez-Morlanes, P. Castell, V. Martínez-Nogués, M. T. Martinez, P. J. Alonso and J. A. Puértolas, Effects of gamma-irradiation on UHMWPE/MWNT nanocomposites, Compos. Sci. Technol., 2011, 71, 282-288.
53 J. Shi, T. Wu, K. Teng, W. Wang, M. Shan, Z. Xu, H. Lv and H. Deng, Simultaneous electrospinning and spraying toward branch-like nanofibrous membranes functionalised with carboxylated MWCNTs for dye removal, Mater. Lett., 2016, 166, 26-29.

54 M. Bartošík, T. Doneux, Z. Pechan, V. Ostatná and E. Paleček, Encyclopedia of Analytical Chemistry, John Wiley \& Sons, Ltd, 2006.

55 E. Barsoukov, R. Macdonald, I. D. Raistrick and D. R. Franceschetti, Impedance Spectroscopy: Theory, Experiment, and Applications, John Wiley \& Sons, 2nd edn, 2005.

56 S. S. Abdel-Rehim, K. F. Khaled and N. S. Abd-Elshafi, Electrochemical frequency modulation as a new technique for monitoring corrosion inhibition of iron in acid media by new thiourea derivative, Electrochim. Acta, 2006, 51, 3269-3277.

57 A. J. Bard and L. R. Faulkner, Electrochemical Methods: Fundamentals and Applications, Wiley, 2000.

58 M. G. Hosseini and M. Abdolmaleki, Synthesis and characterization of porous nanostructured $\mathrm{Ni} / \mathrm{PdNi}$ electrode towards electrooxidation of borohydride, Int. J. Hydrogen Energy, 2013, 38, 5449-5456.

59 M. G. Hosseini and E. Shahryari, Synthesis, Characterization and Electrochemical Study of Graphene Oxide-Multi Walled Carbon Nanotube-Manganese Oxide-Polyaniline-Electrode as Supercapacitor, J. Mater. Sci. Technol., 2016, 32, 763-773.

60 A. Imani, G. Farzi and A. Ltaief, Facile synthesis and characterization of polypyrrole-multiwalled carbon nanotubes by in situ oxidative polymerization, Int. Nano Lett., 2013, 3, 52.

61 T. I. W. Schnoor, U. Vainio, L.-H. Shao, E. T. Lilleodden, M. Müller, A. Schreyer, K. Schulte and B. Fiedler, Nanostructured MWCNT/Polypyrrole Actuators with Anisotropic Strain Response, Advanced Engineering Materials, 2016, 18, 597-607.

62 N. Sankar, M. N. Reddy and R. K. Prasad, Carbon nanotubes dispersed polymer nanocomposites: mechanical, electrical, thermal properties and surface morphology, Bull. Mater. Sci., 2016, 39, 47-55.

$63 \mathrm{~J} . \mathrm{W}$. Lee and Y.-T. Yoo, Preparation and performance of IPMC actuators with electrospun Nafion ${ }^{\circledR}-M W N T$ composite electrodes, Sens. Actuators, B, 2011, 159, 103-111.

64 J.-W. Lee, J.-H. Kim, Y. S. Chun, Y. T. Yoo and S. M. Hong, The performance of Nafion-based IPMC actuators containing polypyrrole/alumina composite fillers, Macromol. Res., 2009, 17, 1032-1038.

65 Q. S. He, X. Q. Zhang, M. Yu and Z. D. Dai, Effects of the Polypyrrole/Silica on the Output Force of Ionic PolymerMetal Composite, Adv. Mater. Res., 2013, 560-565.

66 K. Kaneto, Research Trends of Soft Actuators based on Electroactive Polymers and Conducting Polymers, J. Phys.: Conf. Ser., 2016, 704, 012004. 